This item was submitted to Loughborough's Research Repository by the author.

Items in Figshare are protected by copyright, with all rights reserved, unless otherwise indicated.

\title{
Uniformly accelerating black holes in a de Sitter universe
}

PLEASE CITE THE PUBLISHED VERSION

LICENCE

CC BY-NC-ND 4.0

\section{REPOSITORY RECORD}

Podolsky, J., and J.B. Griffiths. 2019. "Uniformly Accelerating Black Holes in a De Sitter Universe”. figshare. https://hdl.handle.net/2134/771. 


\title{
Uniformly accelerating black holes in a de Sitter universe
}

\author{
J. Podolský* \\ Institute of Theoretical Physics, Charles University, \\ V Holešovičkách 2, 18000 Prague 8, Czech Republic. \\ and \\ J. B. Griffiths ${ }^{\dagger}$ \\ Department of Mathematical Sciences, Loughborough University, \\ Loughborough, Leicestershire LE11 3TU, U.K.
}

October 31, 2000

\begin{abstract}
A class of exact solutions of Einstein's equations is analysed which describes uniformly accelerating charged black holes in an asymptotically de Sitter universe. This is a generalisation of the C-metric which includes a cosmological constant. The physical interpretation of the solutions is facilitated by the introduction of a new coordinate system for de Sitter space which is adapted to accelerating observers in this background. The solutions considered reduce to this form of the de Sitter metric when the mass and charge of the black holes vanish.
\end{abstract}

\section{Introduction}

It has recently been suggested [1] that the pair creation of black holes is possible in a background with a cosmological constant as this supplies the necessary negative potential energy. To investigate this process, an exact solution of Einstein's equations is required which represents a pair of black holes which accelerate away from each other in a de Sitter background. In fact, a general class of such solutions is available [2] (and used in [1]) which includes mass and charge parameters as well as a cosmological constant $\Lambda$. When $\Lambda=0$, these solutions include the well-known C-metric which describes two black holes which uniformly accelerate in a Minkowski background under the action of conical singularities [3]. However, for $\Lambda \neq 0$, the physical, geometrical and global properties of the space-time [2] has not been investigated thoroughly even at the classical level.

It is the purpose of the present paper to provide a physical interpretation for this family of exact solutions. We will show that indeed it represents (possibly charged) black holes uniformly accelerating in a de Sitter universe. This interpretation is obtained after first introducing a new coordinate system which is adapted to the motion of (two) uniformly accelerating test particles in de Sitter space. We will then show that, for small mass and charge parameters, some of the Plebanski-Demianski solutions [2] can be regarded as perturbations of de Sitter space in these coordinates. This enables the physical meaning of the parameters to be determined.

${ }^{*}$ E-mail: Podolsky@mbox.troja.mff.cuni.cz

${ }^{\dagger}$ E-mail: J.B.Griffiths@Lboro.ac.uk 


\section{Uniformly accelerating observers in de Sitter space}

It is well-known that de Sitter space can be represented as a 4-hyperboloid

$$
-Z_{0}^{2}+Z_{1}^{2}+Z_{2}^{2}+Z_{3}^{2}+Z_{4}^{2}=a^{2} \equiv 3 / \Lambda
$$

in a 5-dimensional Minkowski space. In the familiar parametrization by static coordinates,

$$
\begin{aligned}
& Z_{0}=\sqrt{a^{2}-R^{2}} \sinh (T / a), \\
& Z_{1}= \pm \sqrt{a^{2}-R^{2}} \cosh (T / a), \\
& Z_{4}=R \cos \Theta, \\
& Z_{2}=R \sin \Theta \cos \Phi, \\
& Z_{3}=R \sin \Theta \sin \Phi,
\end{aligned}
$$

the metric is,

$$
\mathrm{d} s^{2}=\frac{\mathrm{d} R^{2}}{1-\frac{R^{2}}{a^{2}}}+R^{2}\left(\mathrm{~d} \Theta^{2}+\sin ^{2} \Theta \mathrm{d} \Phi^{2}\right)-\left(1-\frac{R^{2}}{a^{2}}\right) \mathrm{d} T^{2},
$$

with $R \in[0, a], T \in(-\infty, \infty), \Theta \in[0, \pi], \Phi \in[0,2 \pi)$. The two maps given by (2) cover two causally disconnected areas $Z_{1}>0$ and $Z_{1}<0$ of the manifold.

Now, let us consider the timelike worldlines $x^{\mu}(\tau)$ :

$$
R=R_{0}, \Theta=\Theta_{0}, \Phi=\Phi_{0}, T=a \tau / \sqrt{a^{2}-R_{0}^{2}},
$$

where $R_{0}, \Theta_{0}$ and $\Phi_{0}$ are constants. The 4 -velocity is $u^{\mu}=\left(0,0,0, a / \sqrt{a^{2}-R_{0}^{2}}\right)$, and the 4-acceleration is $\dot{u}^{\mu}=\mathrm{D} u^{\mu} / \mathrm{d} \tau \equiv u_{; \nu}^{\mu} u^{\nu}=\left(-R_{0} / a^{2}, 0,0,0\right)$. Obviously, $\tau$ is the proper time since $u_{\mu} u^{\mu}=-1$, and the 4 -acceleration is constant with modulus

$$
|A| \equiv\left|\dot{u}^{\mu}\right|=\frac{R_{0}}{a \sqrt{a^{2}-R_{0}^{2}}} .
$$

Since $u_{\mu} \dot{u}^{\mu}=0$, this constant value of $|A|$ is identical to the modulus of the 3 -acceleration measured in the natural local orthonormal frame of the observer.

The worldlines (4) therefore represent the motion of uniformly accelerating observers in a de Sitter universe. When $R_{0}=0$ the worldline is a geodesic with zero acceleration. On the other hand, when $R_{0}=a$, the acceleration $A$ is unbounded and motion is along the null cosmological horizon. In general, these uniformly accelerated trajectories are given by constant values of $Z_{2}$, $Z_{3}$ and $Z_{4}$ on the de Sitter hyperboloid, and coincide with the orbits of the isometry generated by the Killing vector $\partial_{T}$.

It is also instructive to visualise these accelerated observers in the standard global coordinate representation of de Sitter space:

$$
\begin{aligned}
& Z_{0}=-a \frac{\cos \eta}{\sin \eta}, \\
& Z_{1}=a \frac{\cos \chi}{\sin \eta}, \\
& Z_{4}=a \frac{\sin \chi}{\sin \eta} \cos \Theta \\
& Z_{2}=a \frac{\sin \chi}{\sin \eta} \sin \Theta \cos \Phi, \\
& Z_{3}=a \frac{\sin \chi}{\sin \eta} \sin \Theta \sin \Phi,
\end{aligned}
$$




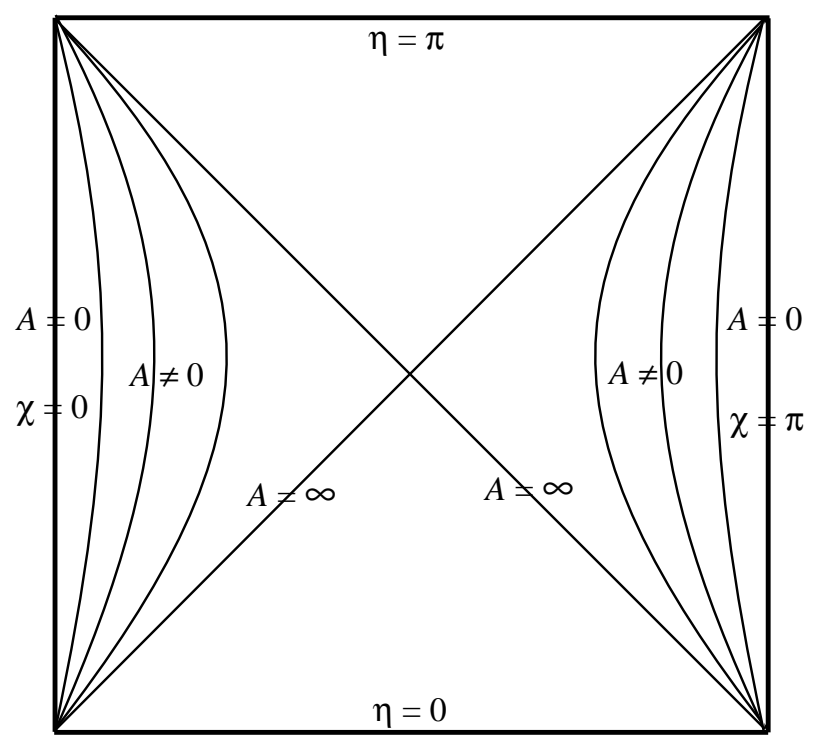

Figure 1: The conformal diagram for de Sitter space indicating trajectories of observers with uniform acceleration $A$. Each point represents a complete 2-sphere.

where $\eta \in[0, \pi], \chi \in[0, \pi]$. With this, the metric is

$$
\mathrm{d} s^{2}=\frac{a^{2}}{\sin ^{2} \eta}\left[\mathrm{d} \chi^{2}+\sin ^{2} \chi\left(\Theta^{2}+\sin ^{2} \Theta \mathrm{d} \Phi^{2}\right)-\mathrm{d} \eta^{2}\right] .
$$

This immediately leads to the familiar conformal diagram of de Sitter space (Fig. 1). Comparing expressions for $Z_{4}$ in (2) and (6), it can be seen that the trajectories of accelerated observers are given by

$$
\sin \chi=\frac{a|A|}{\sqrt{1+a^{2} A^{2}}} \sin \eta
$$

and indicated in Fig. 1.

\section{New accelerating coordinates for de Sitter space}

We now introduce a new coordinate system which is particularly well suited for studying uniformly accelerating point sources in de Sitter space. This is given by the following parametrization of $(1)$

$$
\begin{aligned}
& Z_{0}=\frac{\sqrt{a^{2}-r^{2}} \sinh (T / a)}{\sqrt{1+a^{2} A^{2}}+A r \cos \theta}, \\
& Z_{1}= \pm \frac{\sqrt{a^{2}-r^{2}} \cosh (T / a)}{\sqrt{1+a^{2} A^{2}}+A r \cos \theta}, \\
& Z_{4}=\frac{\sqrt{1+a^{2} A^{2}} r \cos \theta+a^{2} A}{\sqrt{1+a^{2} A^{2}}+A r \cos \theta}, \\
& Z_{2}=\frac{r \sin \theta \cos \Phi}{\sqrt{1+a^{2} A^{2}}+A r \cos \theta}, \\
& Z_{3}=\frac{r \sin \theta \sin \Phi}{\sqrt{1+a^{2} A^{2}}+A r \cos \theta} .
\end{aligned}
$$

With $r \in[0, a], \quad T \in(-\infty, \infty), \quad \theta \in[0, \pi], \quad \Phi \in[0,2 \pi)$, this again covers two causally disconnected areas of the de Sitter manifold. In these coordinates, the de Sitter space is represented 
in the form

$$
\begin{aligned}
\mathrm{d} s^{2}= & \frac{1}{\left[\sqrt{1+a^{2} A^{2}}+A r \cos \theta\right]^{2}}\left\{\frac{\mathrm{d} r^{2}}{1-\frac{r^{2}}{a^{2}}}\right. \\
& \left.+r^{2}\left(\mathrm{~d} \theta^{2}+\sin ^{2} \theta \mathrm{d} \Phi^{2}\right)-\left(1-\frac{r^{2}}{a^{2}}\right) \mathrm{d} T^{2}\right\} .
\end{aligned}
$$

This new static metric is conformal to the standard form (3) to which it reduces when $A=0$. The transformation between (3) and (10) is given by relating $r, \theta$ to $R, \Theta$ as

$$
\begin{aligned}
R^{2}-a^{2} & =\frac{r^{2}-a^{2}}{\left[\sqrt{1+a^{2} A^{2}}+A r \cos \theta\right]^{2}}, \\
R \sin \Theta & =\frac{r \sin \theta}{\sqrt{1+a^{2} A^{2}}+A r \cos \theta}, \\
R \cos \Theta & =\frac{\sqrt{1+a^{2} A^{2}} r \cos \theta+a^{2} A}{\sqrt{1+a^{2} A^{2}}+A r \cos \theta},
\end{aligned}
$$

which can be obtained by comparing (2) with (9).

It is obvious that the origin $r=0$ of the coordinates (10) corresponds to $R_{0}=a^{2}|A| / \sqrt{1+a^{2} A^{2}}$ and $Z_{2}=0=Z_{3}$. Substituting the above value of $R_{0}$ into (5), we conclude that the parameter $A$ in the metric (10) is exactly the value of the acceleration of the corresponding observer. Therefore, the origin $r=0$ of the coordinates in (10) is accelerating in a de Sitter universe with uniform acceleration $A$. This uniformly accelerated trajectory corresponds to the motion of two distinct points on the de Sitter hyperboloid, one in each of the two causally disconnected static regions. When $A>0$ the coordinate singularity $r=0$ is located at $\Theta_{0}=0$ so that $Z_{4}=R_{0}>0$, whereas when $A<0$ it is located at $\Theta_{0}=\pi$ so that $Z_{4}=-R_{0}<0$. It may further be noted that all observers having arbitrary constant values of $r, \theta$ and $\Phi$ also move with uniform acceleration (generally different from $A$ ).

The character of these coordinates can be further understood by expressing them in terms of the global coordinates (6) by

$$
\begin{aligned}
1-\frac{r^{2}}{a^{2}} & =\frac{\sin ^{2} \eta-\sin ^{2} \chi}{\left[\sqrt{1+a^{2} A^{2}} \sin \eta-a A \sin \chi \cos \Theta\right]^{2}}, \\
r \sin \theta & =\frac{a \sin \chi \sin \Theta}{\sqrt{1+a^{2} A^{2}} \sin \eta-a A \sin \chi \cos \Theta}, \\
\tanh \left(\frac{T}{a}\right) & =\mp \frac{\cos \eta}{\cos \chi} .
\end{aligned}
$$

Notice again that $r=0$ implies both (8) and $\Theta=0$ (or $\Theta=\pi$ for $A<0$ ). These coordinates cover the regions I or III according to the two signs of $Z_{1}$ in (9). This is illustrated in the conformal diagram of de Sitter space (Fig. 2). It may be observed that $r=0$ is represented by two worldlines located in regions I and III. The cosmological horizon occurs when $r=a$. The coordinate $r$ in (10) can in fact be extended through this horizon into the nonstatic regions II and IV, where it becomes timelike. However, other coordinate charts are required there since $T \in(-\infty, \infty)$ covers only regions I and III between $r=0$ and the cosmological horizon. These are obtained by replacing $\sqrt{a^{2}-r^{2}}$ in (9) by $\sqrt{r^{2}-a^{2}}$.

Note that the explicit spherical symmetry of the coordinate system has been removed to accommodate a description of uniform acceleration. However, this introduces difficulties in describing the de Sitter timelike and null infinity given by $\eta=0, \pi \quad$ (or $R=\infty$ ) using the coordinates of (10). It may be observed from (11) that points in regions II and IV in Fig. 2 with $R>\sqrt{1+a^{2} A^{2}} / A$ can only be reached for a limited range of the coordinate $\theta$. Moreover, infinity can be reached at a finite value of the timelike coordinate $r$ such that $r \cos \theta=-\sqrt{1+a^{2} A^{2}} / A$ at which the conformal factor in (10) is unbounded. Only in the "equatorial plane" $\theta=\pi / 2$ does $r=\infty$ correspond to $R=\infty$. 


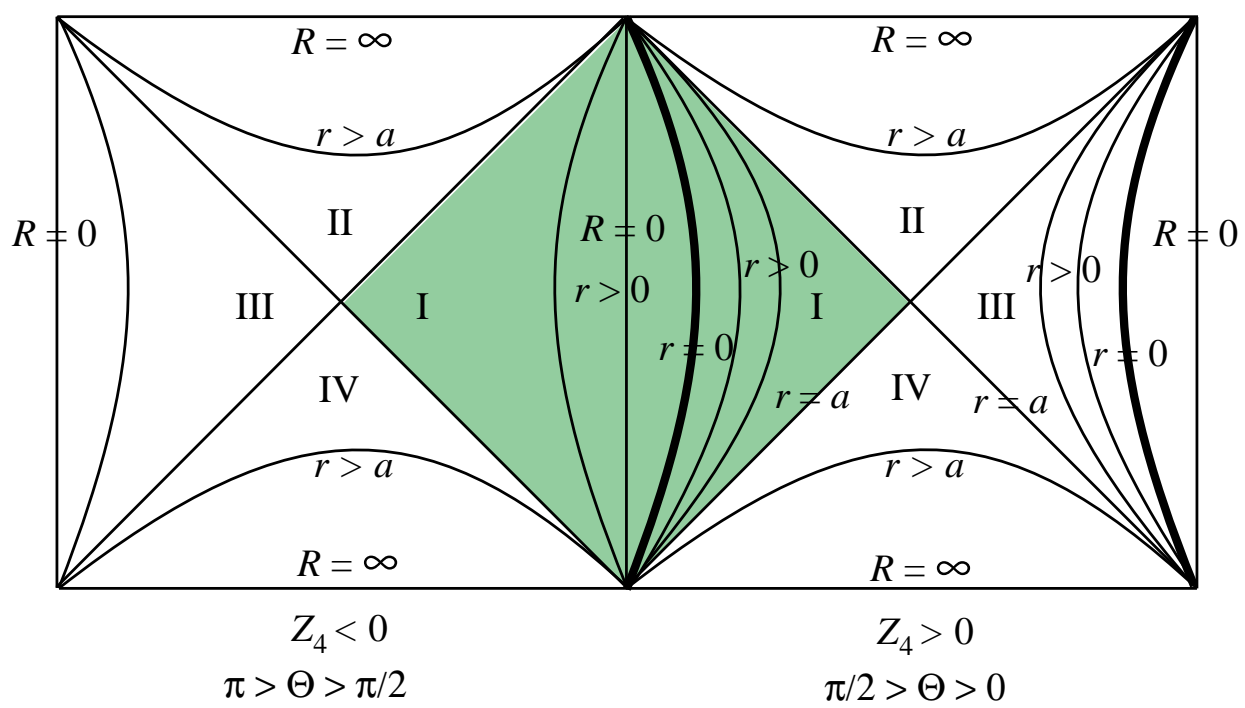

Figure 2: The conformal diagram for de Sitter space such that each point represents a hemisphere. The shaded area indicates the region I covered by the new coordinates of (10) for $0 \leq r \leq a$. The complete space repeats this area in III and includes the regions II and IV beyond the cosmological horizons. Thus $r=0$ represents two uniformly accelerating points on causally disconnected opposite sides of the universe.

\section{The generalised C-metric}

Let us now consider the Einstein space described by the line element

$$
\mathrm{d} s^{2}=\frac{1}{(p+q)^{2}}\left(\frac{\mathrm{d} p^{2}}{\mathcal{P}}+\frac{\mathrm{d} q^{2}}{\mathcal{Q}}+\mathcal{P} \mathrm{d} \sigma^{2}-\mathcal{Q} \mathrm{d} \tau^{2}\right),
$$

where

$$
\begin{aligned}
& \mathcal{P}(p)=A^{2}-p^{2}+2 m p^{3}-e^{2} p^{4} \\
& \mathcal{Q}(q)=-\frac{\Lambda}{3}-A^{2}+q^{2}+2 m q^{3}+e^{2} q^{4}
\end{aligned}
$$

This is contained in the large family of solutions given by Plebanski and Demianski [2] (see equation (6.3), and also [4]-[6]) in which the "rotation" vanishes. We note that the linear terms have been removed in (14) using the shift $p \rightarrow p+c_{0}$ and $q \rightarrow q-c_{0}$, where $c_{0}$ is a constant. Also, the coefficients of the quadratic terms have been set to unity by a rescaling of coordinates. (The possibility of different signs for the quadratic terms has been considered in [7].)

When the cosmological constant in (14) vanishes, (13) reduces to the well-known C-metric which has been interpreted [3] as describing two black holes, each of mass $m$ and charge $e$, which move in opposite directions relative to a Minkowski background with acceleration $A$ under the action of some conical singularities. We will argue below that the metric in the form $(13,14)$ can be considered as the most natural generalisation of the C-metric which includes a cosmological constant $\Lambda$.

Note that in (14) the acceleration parameter $A$ is introduced in a different form to that considered previously (e.g. [1]). We use a different scaling, and also adapt the constant terms so that $\Lambda$ appears explicitly in $\mathcal{Q}$ only. We will demonstrate below that this is more convenient for physical interpretation.

In order to maintain the correct space-time signature, it is necessary that $\mathcal{P}>0$. This places a restriction on the range of $p$. However, there is no restriction on the sign of $\mathcal{Q}$ which may describe both static and nonstatic regions, with horizons occurring when $\mathcal{Q}=0$. 


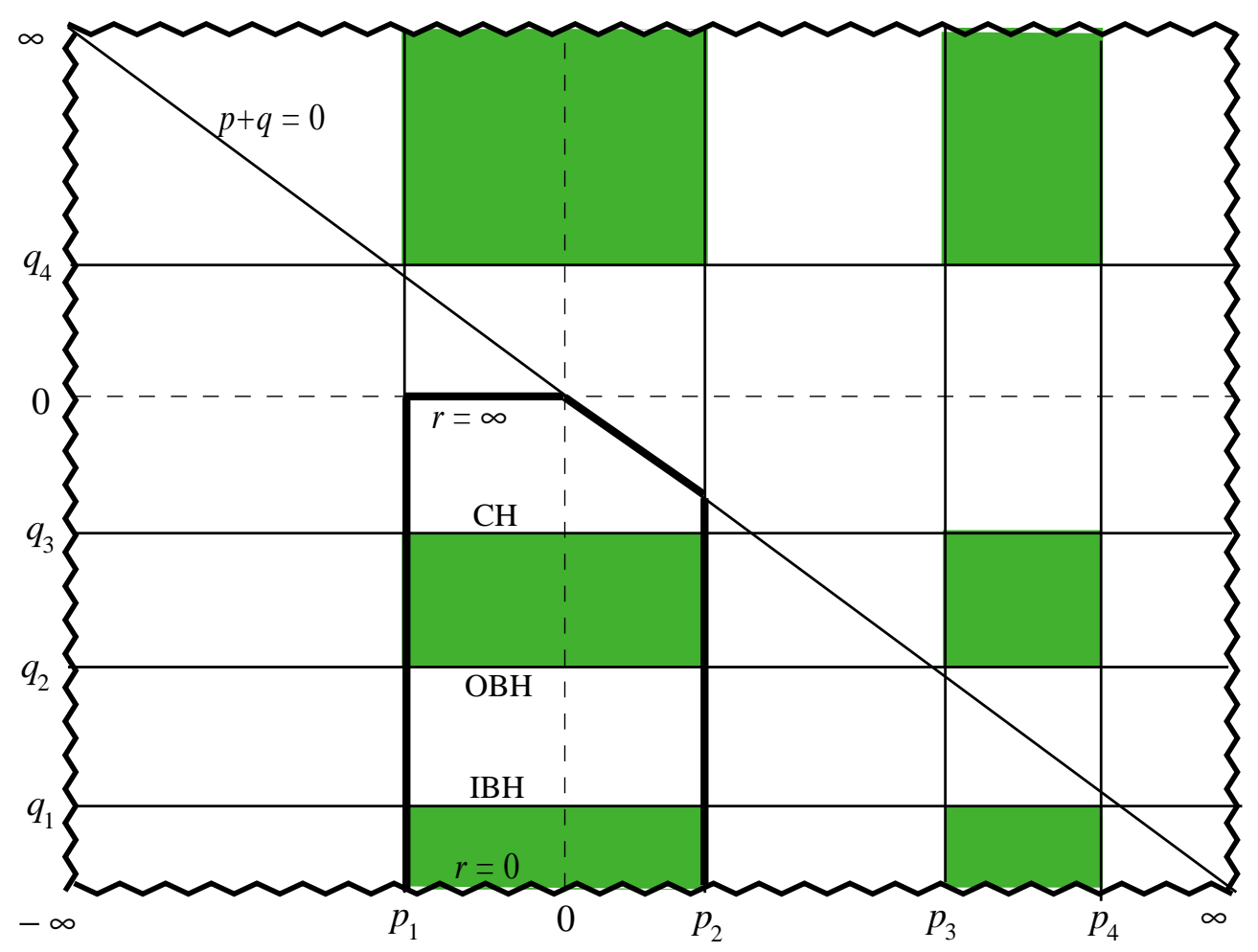

Figure 3: In the full ranges of $p$ and $q$, space-times only occur when $p$ lies between the roots $p_{1}$ and $p_{2}$, or between $p_{3}$ and $p_{4}$. These include six possible static space-time regions which are indicated by the shaded areas. Attention is focussed here on the space-time spanned by $p_{1}<p<p_{2}$ and $q<-p$. This contains two static and two nonstatic regions separated by a cosmological horizon $(\mathrm{CH})$ and inner and outer black hole horizons (IBH and OBH). Apart from a region near conformal infinity, this space-time is also covered by the coordinates $r$ and $\theta$ which span the regions indicated by the area within the bold lines.

Let us assume that there is a finite range of $p$, bounded by roots $p_{1}$ and $p_{2}\left(>p_{1}\right)$, in which $\mathcal{P} \geq 0$. This spans the space-time which we wish to investigate. However, it is convenient to introduce the parameter $\zeta$ by $p=A \zeta$, so that $\zeta_{1} \leq \zeta \leq \zeta_{2}$, where $\zeta_{i}=p_{i} / A$.

Now, let us perform the coordinate transformation

$$
\begin{aligned}
& r=-\sqrt{1+a^{2} A^{2}} / q, \\
& \theta=\int_{\zeta_{1}} \frac{\mathrm{d} \zeta}{\sqrt{1-\zeta^{2}+2 m A \zeta^{3}-e^{2} A^{2} \zeta^{4}}}, \\
& \Phi=A \sigma / c \\
& T=\sqrt{1+a^{2} A^{2}} \tau,
\end{aligned}
$$

where $c$ is a constant which can be specified later. With this, the metric (13) becomes

$$
\begin{aligned}
\mathrm{d} s^{2}= & \frac{1}{\left[\sqrt{1+a^{2} A^{2}}-A r \zeta(\theta)\right]^{2}} \times \\
& \left\{\frac{\mathrm{d} r^{2}}{F(r)}+r^{2}\left(\mathrm{~d} \theta^{2}+G^{2}(\theta) c^{2} \mathrm{~d} \Phi^{2}\right)-F(r) \mathrm{d} T^{2}\right\},
\end{aligned}
$$

where

$$
F(r)=1-\frac{r^{2}}{a^{2}}-\sqrt{1+a^{2} A^{2}} \frac{2 m}{r}+\left(1+a^{2} A^{2}\right) \frac{e^{2}}{r^{2}},
$$




$$
G^{2}(\theta)=1-\zeta^{2}(\theta)+2 m A \zeta^{3}(\theta)-e^{2} A^{2} \zeta^{4}(\theta)
$$

and $\zeta(\theta)$ is the inverse function of $\theta(\zeta)$ given by the integral in (15). It may be seen that, either when $A=0$ or when both $m=0$ and $e=0$, we have $\zeta(\theta)=-\cos \theta$ so that $G(\theta)=\sin \theta$. Otherwise these can be expressed in terms of Jacobian elliptic functions. For example, when $G^{2}(\zeta)$ has four distinct real roots $\zeta_{1}<\zeta_{2}<\zeta_{3}<\zeta_{4}$,

$$
\zeta(\theta)=\frac{\zeta_{1}\left(\zeta_{4}-\zeta_{2}\right)+\zeta_{4}\left(\zeta_{2}-\zeta_{1}\right) \operatorname{sn}^{2}(n \theta \mid k)}{\left(\zeta_{4}-\zeta_{2}\right)+\left(\zeta_{2}-\zeta_{1}\right) \operatorname{sn}^{2}(n \theta \mid k)},
$$

where sn is the Jacobian elliptic function (see [8]), with

$$
n=\frac{1}{2} \sqrt{\left(\zeta_{3}-\zeta_{1}\right)\left(\zeta_{4}-\zeta_{2}\right)}, \quad k=\frac{\left(\zeta_{2}-\zeta_{1}\right)\left(\zeta_{4}-\zeta_{3}\right)}{\left(\zeta_{3}-\zeta_{1}\right)\left(\zeta_{4}-\zeta_{2}\right)}
$$

The possible ranges of the coordinates $p$ and $q$ are illustrated in Fig. 3. Assuming that $\mathcal{P}(p)$ and $\mathcal{Q}(q)$ both have four real roots, there are six possible static space-time regions for all permitted values. These are bounded by horizons (roots of $\mathcal{Q}$ ) or coordinate singularities (roots of $\mathcal{P}$ ). Attention is concentrated here on the space-time for which $p_{1}<p<p_{2}$ and $p+q<0$. This covers two static and two nonstatic regions separated by a cosmological horizon and by inner and outer black hole horizons. However, these regions are not entirely covered by the coordinates $r$ and $\theta$, as is also illustrated.

It may be observed that, although the $r, \theta$ coordinates appropriately describe the black hole regions and even the cosmological horizon, they are not well suited to investigating timelike or null infinity. The restriction $r>0$ corresponds to $q<0$ (15), and it is immediately obvious from Fig. 3 that the space-time boundary $p+q=0$ (where the conformal factor in (16) is unbounded) can only be reached for part of the range of $\theta$, and then for finite values of $r$.

It may also be observed that the region $p_{3}<p<p_{4}, p+q>0$ in Fig. 3 represents the same space-time with the replacements $p \rightarrow-p, q \rightarrow-q$ and $m \rightarrow-m$.

\section{Physical interpretation}

It is obvious that, when $A=0$ and $c=1,(16)$ immediately reduces to the familiar form of the Reissner-Nordstrøm-de Sitter black hole solution in which the parameters $m$ and $e$ have the usual interpretation and the curvature singularity is located at $r=0$. Moreover, when $A \neq 0, c=1$ and $m=0=e$, the metric (16) is identical to (10) in which $r=0$ corresponds to two uniformly accelerating points relative to the de Sitter background. When $m$ and $e$ are small, (16) can naturally be regarded as a perturbation of (10). In this way, the metric (16) can be interpreted as describing a pair of charged black holes uniformly accelerating in a de Sitter universe. However, for a finite acceleration $A$, it follows from (17) that the effective mass and charge of each black hole are given respectively by $\sqrt{1+a^{2} A^{2}} m$ and $\sqrt{1+a^{2} A^{2}} e$.

The space-times considered here possess the "boost" and "rotation" symmetries associated with the Killing vectors $\partial_{T}$ and $\partial_{\Phi}$. In the 5-dimensional representation (1) of the de Sitter background, these are given by $Z_{0} \partial_{Z_{1}}+Z_{1} \partial_{Z_{0}}$ and $Z_{2} \partial_{Z_{3}}-Z_{3} \partial_{Z_{2}}$ which are the analogues of those described in [10] for $\Lambda=0$.

Killing horizons (where the norm of the Killing vector $\partial_{T}$ vanishes) between static and radiative regions of these space-times occur when $F=0$. Generally, $F(r)$ can have up to four real roots, one of which must be negative. However, taking $r \geq 0, F$ can have at most three positive real roots. In this case, the space-time will include the familiar inner and outer black hole and cosmological horizons, although it may be noted that their geometrical properties are altered by the presence of acceleration. Cases describing accelerating extreme black holes and naked singularities (in which the roots are repeated or complex) are also included in (16) and (17) for specific ranges of the parameters. 
When the acceleration vanishes, the conformal diagrams of all the possible (spherically symmetric) cases are known (see [9]). It is interesting that these diagrams also describe the global structure of (16) in the plane $\zeta=0$ "orthogonal" to the direction of the acceleration, even in the case when $A \neq 0$. On this plane $r=\infty$ corresponds to de Sitter-like infinity.

For nonzero acceleration, the complete global structure is very complicated. For small $m$ and $e$, these space-times can be considered as perturbations of a de Sitter universe as illustrated in Figs 1 and 2. In this context, these figures should be regarded as useful schematic pictures rather than conformal diagrams since $r=0$ is now a curvature singularity and other horizons occur. In particular, it may be observed that the space-time describes the motion of two black holes accelerating in the de Sitter background.

As pointed out in section 3, for some range of $\theta$, the de Sitter infinity is reached at a finite value of the coordinate $r$ (which is timelike in this region) given by $r \zeta(\theta)=\sqrt{1+a^{2} A^{2}} / A$. For this value, the conformal factor in (16) is unbounded (corresponding to $p+q=0$ in (13)). For the coordinates of (16), this apparent angular dependence at infinity is clearly illustrated in Fig. 3 as discussed in section 4 . However, it can be shown that the proper time required to reach this boundary is always unbounded.

Let us finally investigate the nature of the singularities at $p=p_{1}=A \zeta_{1}$ and $p=p_{2}=A \zeta_{2}$, where $\mathcal{P}\left(p_{i}\right)=0$, i.e. $G=0$. It follows from (15) that the singularity at $\zeta=\zeta_{1}$ corresponds to $\theta=0$, and $\zeta=\zeta_{2}$ to $\theta=2 K$, where $K$ is the "quarter period" (the complete elliptic integral of the first kind related to (15)) which is $\pi / 2$ when either $A=0$ or $m=0=e$.

Consider 2-dimensional spacelike surfaces on which $r$ and $T$ are constants and $\theta \in[0,2 K]$, $\Phi \in[0,2 \pi)$. By comparing the circumference of a small circle (fixed $\theta$ ) around the pole $\theta=0$ with its "radius" (segment with fixed $\Phi$ ), we find that in general there is a deficit angle

$$
\begin{aligned}
\delta_{1} & =2 \pi\left[1-\lim _{\theta \rightarrow 0} \frac{c G(\theta)}{\theta}\right]=2 \pi\left[1-c G^{\prime}(0)\right] \\
& =2 \pi\left[1+c\left(\zeta_{1}-3 m A \zeta_{1}^{2}+2 e^{2} A^{2} \zeta_{1}^{3}\right)\right] .
\end{aligned}
$$

This is finite and also independent of $r$ and $T$. Thus, the singularity $p=p_{1}$ represents a cosmic string of constant tension along the "semi-axis" $\theta=0$. However, for any value of $m, e, A$ and $\Lambda$, this axis can always be made regular by putting $c=1 / G^{\prime}(0)$. In particular, if $A=0$ or if $m$ and $e$ are both zero, $G=\sin \theta$ and the axis is regular when $c=1$ as required for the spherically symmetric Reissner-Nordstrøm-de Sitter space-time or for the de Sitter universe in accelerating coordinates.

For the alternative pole $\theta=2 K$, the deficit angle of a cosmic string in the opposite direction is given by

$$
\begin{aligned}
\delta_{2} & =2 \pi\left[1-\lim _{\theta \rightarrow 2 K} \frac{c G(\theta)}{2 K-\theta}\right]=2 \pi\left[1+c G^{\prime}(2 K)\right] \\
& =2 \pi\left[1-c\left(\zeta_{2}-3 m A \zeta_{2}^{2}+2 e^{2} A^{2} \zeta_{2}^{3}\right)\right] .
\end{aligned}
$$

This could be removed by setting $c=-1 / G^{\prime}(2 K)$. However, it is not possible in general to remove the strings in both directions simultaneously unless $G^{\prime}(0)=-G^{\prime}(2 K)$. This condition is identically satisfied for $m=0=e$ or $A=0$ with $c=1$. However, for small $m A$, a linear perturbation about $\zeta_{1}=-1$ and $\zeta_{2}=1$ indicates that the two strings cannot be removed simultaneously. It follows that a physically reasonable $(e<m)$ accelerating black hole in a de Sitter background must be connected to at least one conical singularity which may be considered to "cause" the acceleration. This has been observed previously [1] using a less appropriate coordinate system.

We have already argued that, relative to a de Sitter background, the space-times contain two accelerating black holes. These are connected by at least one string, localised at $\theta=0$ and/or $\theta=2 K$. Since each point in Fig. 1 represents a 2-dimensional compact subspace spanned by 
$\theta$ and $\Phi$, the strings may be located on either of the two "antipodal" points on all of these "2-spheres" and thus connects one black hole to the other.

In the interpretation [11] of the C-metric with $\Lambda=0$, it was convenient to express the metric in the Weyl form. Here it is also possible to transform (16) to the analogous Lewis-Papapetrou form, at least within each static region, by putting $\rho=\rho_{0}+\int \mathrm{d} r / r \sqrt{F(r)}$, where $\rho_{0}$ is a suitable constant. However, since this applies only in disconnected regions of the space-time, it appears to be less convenient for a global interpretation of these solutions. In fact, the structure of the two cases with zero or nonzero $\Lambda$ are very different. It is therefore not surprising that there is no simple reduction of (16) to the C-metric as $\Lambda \rightarrow 0$. Nevertheless, it is again possible to perform a coordinate transformation

$$
\begin{aligned}
\rho & =\frac{1}{2} b\left(\xi^{2}+t^{2}-\psi^{2}\right), \\
\theta-K & =b \xi \sqrt{\psi^{2}-t^{2}}, \\
T & = \pm a \operatorname{arctanh}(t / \psi),
\end{aligned}
$$

$b=$ const., with which the metric becomes

$$
\begin{aligned}
\mathrm{d} s^{2}= & \mathcal{F} \mathrm{d} \xi^{2}+\mathcal{G} \mathrm{d} \Phi^{2} \\
& +\mathcal{F} \frac{(\psi \mathrm{d} \psi-t \mathrm{~d} t)^{2}}{\psi^{2}-t^{2}}-\mathcal{H} \frac{(t \mathrm{~d} \psi-\psi \mathrm{d} t)^{2}}{\left(\psi^{2}-t^{2}\right)^{2}}
\end{aligned}
$$

where

$$
\begin{aligned}
\mathcal{F} & =\frac{b^{2} r^{2}(\rho)\left(\xi^{2}+\psi^{2}-t^{2}\right)}{\left[\sqrt{1+a^{2} A^{2}}-A r(\rho) \zeta(\theta)\right]^{2}}, \\
\mathcal{G} & =\frac{c^{2} r^{2}(\rho) G^{2}(\theta)}{\left[\sqrt{1+a^{2} A^{2}}-A r(\rho) \zeta(\theta)\right]^{2}}, \\
\mathcal{H} & =\frac{a^{2} F(r(\rho))}{\left[\sqrt{1+a^{2} A^{2}}-A r(\rho) \zeta(\theta)\right]^{2}} .
\end{aligned}
$$

The form (22) exhibits the "boost-rotational" symmetry explicitly. It is similar to the analogous form for $\Lambda=0$. It also enables a natural extension of the static Lewis-Papapetrou metric to regions with $|t|>|\psi|$.

Note finally that some interesting new features occur when $\Lambda \neq 0$. These arise since the universe is now closed and expanding. For example, a cosmic string starting at one black hole must extend to the other. Then, proceeding from the opposite pole of the second black hole, a second (possible) string eventually returns to the first black hole from the opposite direction. As a second observation, we note that the acceleration of any object can have both positive and negative signs simultaneously. This arises since a point moving away in one direction is also approaching from the opposite side of the universe.

\section{Acknowledgements}

We are grateful to J. Bičák for some useful suggestions. This work was supported by a visiting fellowship from the Royal Society and, in part, by the grant GACR-202/99/0261 of the Czech Republic and GAUK 141/2000 of Charles University.

\section{References}

[1] Mann, R. B. and Ross, S. F. (1995). Phys. Rev. D 52, 2254.

[2] Plebanski, J. F. and Demianski, M. (1976), Ann. Phys. (NY) 98, 98. 
[3] Kinnersley, W. and Walker, M. (1970). Phys. Rev. D 2, 1359.

[4] Carter, B. (1968). Commun. Math. Phys. 10280.

[5] Debever, R. (1971). Bull. Soc. Math. Belg. 23, 360.

[6] Kramer, D., Stephani, H., MacCallum, M. and Herlt, E. (1980). Exact solutions of Einstein's field equations, (Cambridge University Press).

[7] Mann, R. B. (1997). Class. Quantum Grav. 14, L109.

[8] Abramowitz, M. and Stegun, I. A. (1965). Handbook of Mathematical Functions, (Dover Publications).

[9] Brill, D. R. and Hayward, S. A. (1994). Class. Quantum Grav., 11, 359.

[10] Bičák, J. and Schmidt, B. (1989). Phys. Rev. D 40, 1827.

[11] Bonnor, W.B. (1983). Gen. Rel. Grav. 15, 535. 